\title{
BMJ Open Examining the effects of enhanced provider-patient communication on postoperative tonsillectomy pain: protocol of a randomised controlled trial performed by nurses in daily clinical care
}

Liesbeth M van Vliet, ${ }^{1}$ Sandra van Dulmen, ${ }^{1,2,3}$ Bram Thiel, ${ }^{4}$ Gerard W van Deelen, ${ }^{5}$ Stephanie Immerzeel, ${ }^{1}$ Marc B Godfried, ${ }^{4}$ Jozien M Bensing ${ }^{1}$

To cite: van Vliet LM, van Dulmen S, Thiel B, et al. Examining the effects of enhanced provider-patient communication on postoperative tonsillectomy pain: protocol of a randomised controlled trial performed by nurses in daily clinical care. BMJ Open 2017;7:e015505. doi:10.1136/ bmjopen-2016-015505

- Prepublication history for this paper is available online. To view these files, please visit the journal online (http://dx.doi. org/10.1136/bmjopen-2016015505).

Received 9 December 2016 Revised 25 September 2017 Accepted 26 September 2017

CrossMark

For numbered affiliations see end of article.

Correspondence to Dr Liesbeth M van Vliet; I.vanvliet@nivel.nl

\section{ABSTRACT}

Introduction Placebo effects (true biopsychological effects not attributable to the active ingredients of medical technical interventions) can be attributed to several mechanisms, such as expectancy manipulation and empathy manipulation elicited by a provider's communication. So far, effects have primarily been shown in laboratory settings. The aim of this study is to determine the separate and combined effects of expectancy manipulation and empathy manipulation during preoperative and postoperative tonsillectomy analgesia care on clinical adult patients' outcomes.

Methods and analysis Using a two-by-two randomised controlled trial, 128 adult tonsillectomy patients will be randomly assigned to one out of four conditions differing in the level of expectancy manipulation (standard vs enhanced) and empathy manipulation (standard vs enhanced). Day care ward nurses are trained to deliver the intervention, while patients are treated via the standard analgesia protocol and hospital routines. The primary outcome, perceived pain, is measured via hospital routine by a Numeric Rating Scale, and additional prehospitalisation, perihospitalisation and posthospitalisation questionnaires are completed (until day 3 , ie, 2 days after the operation). The manipulation is checked using audio recordings of nurse-patient interactions.

Ethics and dissemination Although communication is manipulated, the manipulations do not cross norms or values of acceptable behaviour. Standard medical care is provided. The ethical committee of the UMC Utrecht and the local OLVG hospital committee approved the study. Results will be published via (inter)national peer-reviewed journals and a lay publication.

Trial registration number NTR5994; Pre-results.

\section{INTRODUCTION}

In clinical care, patients' outcomes are influenced not only by the active ingredients of

\section{Strengths and limitations of this study}

- This study is a randomised controlled trial on the (placebo) effects of communication on top of standard medical care, building the evidence base of communication.

- This study is conducted in clinical care opposed to a laboratory setting; strenghtening the results' generalizability.

- The success of the intervention will depend on the ability of nurses to carry out the different communication styles successfully.

drugs or other medical technical interventions but also by the context in which care is delivered. Such biopsychosocial effects on patients' outcomes that are not attributable to the active ingredients of treatments or interventions are called 'non-specific' or 'placebo effects'. 12 They are real and robust, occurring on top of natural history and regression to the mean, ${ }^{3}$ and can be observed alongside 'sham treatments' as well as 'real treatments'. 4

Several mechanisms underlie the generation of placebo effects on patient outcomes such as pain. ${ }^{5}$ A well-understood mechanism is the manipulation of expectations. According to a recent systematic review of our research group, manipulating patients' expectations seems capable of influencing clinical patients' pain perceptions. ${ }^{6}$ For example, the verbal suggestion that a drug is an active pain killer is more effective than receiving the same dosage medication without such a suggestion. ${ }^{7}$ Manipulating expectations also contribute to the recent described positive effects of open-label 
placebos (inert treatments being described as such). ${ }^{8} \mathrm{~A}$ less well understood mechanism is the communication of empathy in healthcare professional-patient encounters. Only few scholars have pointed out the potential role of the professional-patient relationship in explaining placebo effects. ${ }^{9-11}$ In our systematic review, we found that empathy had a less strong effect on pain compared with expectations, ${ }^{6}$ but studies used different empathy operationalisation and empathy was often manipulated together with other elements of the clinical encounter, making it difficult to draw strong conclusions from empathy. ${ }^{6}$

When looking at these mechanisms, it seems reasonable that healthcare professionals' communication can influence them, and can thus produce placebo effects. Until recently, however, the entities of communication and placebo effects have hardly been integrated. ${ }^{11}$ Communication is traditionally associated with 'art, not science' and placebos with 'evidence-based medicine' in which their effects are typically ruled out by the study design of randomised controlled trials (RCTs).

The tide is changing. A landmark study led by Kaptchuk et $a l^{10}$ found that placebo acupuncture delivered with high outcome expectations and an empathic approach led to statistically and clinically significant improvements in the functioning of patients with irritable bowel syndrome compared with placebo acupuncture delivered without expectations and empathy. The distinct and combined effects of both mechanism and the effects alongside an active treatment remain, however, unknown from this study.

Our research group has started to unravel the potential separate and combined effects of both expectancy manipulation and empathy manipulation in highly controlled settings. Using scripted video vignettes and role-play studies, we found that expectancy mainly influences cognitive outcomes (eg, expected treatment effect) and empathy mainly influences affective outcomes (eg, anxiety). The largest positive effects were found when the two elements were combined and a physician raised high expectations, meanwhile communicating in a warm, empathic manner. ${ }^{12}{ }^{13}$ However, whether these distinct and combined effects also translate to the clinical setting, alongside an active intervention, remains, as yet, an unanswered question. Answering this question is important, as it will provide insight into how specific communication elements can influence specific health outcomes.

\section{Study objective}

This study, therefore, aims to disentangle the role of communication in eliciting placebo effects in the clinical setting within the context of standard medical care. More specifically, the objective is to determine the separate and combined effects of expectancy manipulation (standard vs enhanced) and empathy manipulation (standard vs enhanced) during preoperative and postoperative tonsillectomy analgesia care on clinical adult patients' outcomes (main outcome measure is pain perception).
This will be studied using a two-by-two RCT design. By following this approach, the evidence base on the effects of expressed outcome expectancy and conveyed empathy will be built in clinical care.

Accompanying the study objective, the goals of this study are in subsequent order:

1. To examine whether adult patients following tonsillectomy in the enhanced outcome expectancy condition will experience less pain (and other outcomes) compared with patients in the standard condition.

2. To examine whether adult patients following tonsillectomy in the enhanced empathy communication condition will experience less pain (and other outcomes) compared with patients in the standard empathy communication condition.

3. To examine the interaction effects of the different levels of outcome expectancy and empathy on adult patients' experiences of pain (and other outcomes).

\section{METHODS AND ANALYSIS Design and setting}

A four-arm (two-by-two design) single-blind RCT will be conducted at the day care nursing wards on two locations of a Dutch general hospital (OLVG Amsterdam), in which adult tonsillectomy patients are preoperatively and postoperatively monitored and treated by nurses. Patients will be randomly assigned to one out of four arms, which vary in the induction of expectations (standard vs enhanced), and (the level of) nurses' communication of empathy (standard vs enhanced). Depending on the patient's allocation, nurses will express a standard or enhanced outcome expectation of patients' pain, and provide care in a standard or enhanced empathic manner. See figure 1 for the study design. All patients will be treated according to the usual analgesic treatment protocol and daily routine care of the hospital. Recruitment started in August 2016 and will presumably continue until early 2018.

\section{Patients}

This study focuses on adult tonsillectomy patients. This population was carefully selected as it is a homogeneous population. These patients are young adults, generally aged 18-35 years, without complex comorbidity (American Society of Anesthesiologists classification 1) and lack a history of chronic pain. Moreover, tonsillectomy is generally accepted as a strong confound nociceptive trigger resulting into high levels of postoperative pain

\begin{tabular}{lccc}
\hline & & \multicolumn{2}{c}{ Expectancy } \\
& & enhanced & standard \\
\hline Empathy & enhanced & Group 1 & Group 2 \\
\cline { 2 - 2 } & standard & Group 3 & Group 4 \\
\hline
\end{tabular}

Figure 1 Study design. 
that only lasts for a relatively short period of time (1-2 weeks).

\section{Inclusion criteria}

In order to be eligible to participate in this study, a patient must meet the following criteria:

- scheduled for tonsillectomy in day care,

- $\geq 18$ years of age,

- speaking and understanding of the Dutch language,

- having mental capacity.

\section{Exclusion criteria}

A potential patient who meets the following criteria will be excluded from participation in this study.

At study start (during inclusion process):

- not scheduled for tonsillectomy in daycare,

- $<18$ years of age,

- not speaking and understanding of the Dutch language,

- lacking mental capacity (cognitive decline, dementia).

During the course of the study:

- patients who experience a postoperative bleeding will be excluded.

- The healthcare professionals involved and research team can decide to withdraw a patient from the study for urgent medical reasons (eg, if patients are not discharged on the day of operation due to complications).

If a patient drops out, data until exclusion will be included in the analyses unless the patient objects to this (this will be asked on exclusion).

\section{Sample size}

The sample size calculation is based on the primary outcome, that is, pain. This calculation is based on a previous similar study, ${ }^{7}$ in which an open versus hidden administration of analgesic showed a difference of 1.2 and a total variance of 2.18. Based on a power of 0.80 and alpha of 0.05 , and including an interaction effect (with a within variance of 1.92), this results in a needed sample size of 32 patients per arm and $4 \times 32=128$ patients in total.

\section{Recruitment}

The recruitment of patients occurs in several steps.

1. Patients are approached for the study while discussing the operation with their consulting ear nose and throat specialist (ie, ENT doctor). All eligible and interested patients are provided with a Patient Information Folder (PIF) and informed consent form. The PIF omits specific study aims but mentions that communication will be manipulated. It is stressed that participation is free of choice and will not affect usual medical care;

2. During the preoperative examination, which is mostly conducted within a few days of the ENT consultation, the anaesthesiology clinician asks whether the patient is informed about the study. If not, they will provide them the PIF and consent form. They will ask whether the patient is interested in participation and whether the research team can call the patient to provide them with more information. This response is noted in the electronic record and transferred to the researchers via an (automated) email;

3. The research team will call the patient, explain the study in more detail and ask the patient to return the completed informed consent form.

Patients who are already planned for surgery when the study opens (the normal time between ENT consultation and the operation is $6-8$ weeks) will be called by involved healthcare practitioners (from the ENT/Anaesthesiology department). They are informed about the study via telephone. In case they are interested, their name and telephone number are transferred to the researchers. The researchers will call the patient, and send them an information letter and consent form which participants can complete and return in case they are willing to participate.

\section{Randomisation}

On providing informed consent, patients are randomised using a random number generator (1:1:1:1 allocation rate). Assignments will be provided via sequentially numbered, opaque, sealed envelopes. A secretary not otherwise involved in the study will open the assignment envelopes.

After patients are randomised, the research team will inform the healthcare professionals about their inclusion. They will insert this information in the medical records of the hospital system (EPIC). Moreover, they will inform the day care administrators and key contact persons about the patients that are scheduled in the upcoming week or days and which condition they are randomised to. On the day of admission, the researchers will ensure that all appropriate systems (eg, the ward lists and the hard copy patient records) are adequately signposted with the patients' condition. We will use colour codes for this to avoid unblinding patients. Only one patient per room is included at any time point.

\section{Intervention}

The intervention consists of a (protocolled) communication manipulation on top of standard analgesic treatment. Nurses at the day care ward will incorporate an (protocolled) expectancy manipulation (standard vs enhanced) related to the effects of the pain medication and empathy manipulation (standard vs enhanced) into their communication. The communication intervention will be provided at all nurse-patient communication moments during patients' stay at the day care ward (preoperation and postoperation, day 1, the day care wards are open between 07:00-18:00 and 6.45-19:00, respectively), and during the nurses' telephone consultation with patients the day post discharge (day 2). In practice, this means that all communication patients receive from day care ward nurses during this time frame will be according to patients' assigned condition. This includes interactions during intake, pain assessments, medication allocation and transferal to the operation theatre and 
from the postanaesthesia care unit (PACU), discharge and all other interactions due to patients' questions or medical need.

All nurses will receive training to ensure their ability to perform the different communication manipulations. We will make use of a professional trainer and comprehensive training protocol including scripts (ie, written examples), video examples and role play. Moreover, posters are placed in the communal spaces for nurses with information about the study, for example, examples of the manipulations and study procedures. Pocket cards with examples of the manipulations are provided. To ensure the communication differs between the four conditions and to minimise carry-over effects (eg, nurses' stressing enhanced expectations display automatically an enhanced empathy style), the four conditions are trained separately and the posters and pockets cards focus on the four different conditions. The importance of the manipulations being successful in order to draw conclusions from the found results is stressed during the training day.

\section{Expectancy manipulation}

In the standard condition, nurses do not aim to create the expectation that the pain medication will work very well. They might use sentences such as 'The medications attempt to reduce your pain ever so slightly', or 'This is your pain medication'.

In the enhanced condition, nurses aim to create the expectation that the pain medication will work very well. They might use sentences such as: 'The medications I am giving you now will lead to a strong decrease of your pain', or 'This pain medication is known for working very well'.

\section{Empathy manipulation}

In the standard condition, nurses aim to create a neutral atmosphere which is standard. They will be trained to (among other behaviour) keep standing when communicating with patients, react with standard empathy to patients' cues and concerns, not explore concerns in detail, to not express extra interest in the patient as a person, to not pay extra attention to not interrupting patients and to not make extra eye contact.

In the enhanced condition, nurses aim to create an atmosphere, which is extra warm and extra friendly. They will be trained to (among other behaviour) introduce themselves properly, sit while communicating with patients, react extra empathically to patients' cues and concerns (verbal and nonverbal) and take their concerns seriously, to show extra interest in the patient as a person, to not interrupt the patient and to make adequate eye contact.

It should be noted that the communication manipulation does not cross important norms or values of acceptable behaviour and that the psychological integrity of patients will not be harmed. An observational study among clinical postsurgical patients showed that nurse-patient interactions are often subject to interruptions related to other tasks, for example, searching for equipment, answering telephone calls or being interrupted by other professionals. ${ }^{14}$ The interruptions and nurses' attempts to address competing demands impact on the time and attention spent with patients. It can, therefore, be assumed that variations in communication are inherently due to clinical encounters. This was also confirmed by field observations conducted by the research team before study start. The aforementioned developed scripts/examples which are used in the training have been commented on by nurses and researchers in a pilot study to ensure they are realistic and do not trespass ethical boundaries and have been finalised in collaboration with involved clinicians.

\section{Standardising of communication}

The communication patients receive from other clinicians involved during their hospitalisation (eg, from the surgical team working in the operation theatre and the clinical team working in the PACU) will be standardised as much as possible. Also, the communication during the preoperative ENT and Anaesthesiology visit will be standardised as much as possible. Involved healthcare professionals will be informed of the study aims and the importance to keep their communication neutral (if possible) (ie, to not provide extra empathy or raise extra expectations about pain) for included patients. This is feasible, as the ENT and anaesthesiology team are involved in the study, and it is uncommon for patients to ask about pain medication during their time at the PACU.

\section{Blinding}

Patients will be blinded to the specific study aims and treatment allocation.

The involved healthcare personnel cannot be blinded. All healthcare personnel involved will receive clear and specific instructions about informing and including patients to preserve experimental control. Besides, all interactions within the study between nurses and patients will be audio recorded to evaluate the fidelity of the communication manipulation.

\section{Study procedure}

After receiving informed consent, the research team will send a baseline questionnaire to the patient. Completion of this questionnaire will be done at home and will take no more than $20 \mathrm{~min}$. Postoperatively (at day care, day 1 ) a short ( $5 \mathrm{~min}$ ) questionnaire is administered. As part of routine care, preoperatively and postoperatively, patients rate their level of pain. At day 2 (the day after discharge), patients will complete another questionnaire about their pain and medication use. At day 3 , a last questionnaire will be completed which will take $20 \mathrm{~min}$. Patients are given the choice between paper and pencil and online completion of questionnaires. This time frame of follow-up is chosen as we expect the effect of the intervention (delivered within a few hours by day care ward nurses solely) to wane within a few days. 
Moreover, the interactions between involved nurses and included patients will be recorded. Nurses will be provided with a portable audio-recording device with microphone. During every visit, the nurses will mention patient's identification number by means of reference, to protect patients' privacy. At day 4 (3 days after discharge), patients will receive a debriefing letter by postal mail which will inform them about the study aims and their assigned condition. If they wish to receive more information, they can contact the research team.

\section{Withdrawal of individual patients}

Patients can leave the study at any time for any reason if they wish to do so without any consequences. The healthcare professionals involved and research team can decide to withdraw a patient from the study for urgent medical reasons (eg, a bleeding after operation). If a patient drops out, the research team is informed of this. Patients will continue to receive standard medical care and communication.

\section{Informed consent nurses}

Nurses involved in the study will be asked to participate as participants using an information sheet and a consent form. They will be asked to complete the consent form and a questionnaire about their background characteristics. We will offer them, at study end, participation in a (accredited) communication training to thank them for their participation in this project.

\section{Outcomes}

\section{Main study outcome}

\section{Pain perception/intensity}

As part of routine care, during hospitalisation and posthospitalisation patients' pain will be assessed on the basis of a Numeric Rating Scale (NRS) (0-10), ranging from 'no pain' to 'worst imaginable pain'. ${ }^{15} 16$ Pain is rated preoperatively at day care ward, at the PACU and postoperatively at day care ward. On study day 2, 1 day after discharge, day care ward nurses will contact the patients by telephone and again assess their pain. On top of this standard routine, pain is assessed at home on day 2 and day 3 (study end).

\section{Secondary study outcome}

In the patient questionnaires, the following secondary outcomes will be assessed:

\section{Pain expectations}

Patients' pain expectations are measured using two items (both measured using a self-created Visual Analogue Scale (VAS): (1) patients' pain expectations for the few days following the operation (VAS ranging from no pain to 'the most intense pain imaginable', ranging from 0 to 10 , adapted from Petersen $e t a l^{17}$ ), and (2) patients' expectations of improvement in pain following receiving pain medication (VAS ranging from ' $0 \%$ improvement' (no improvement) to ' $100 \%$ improvement' (most improvement imaginable), adapted from the Credibility and Expectancy Questionnaire. ${ }^{18}$ These questions will be assessed postoperatively (during hospitalisation).

\section{Overall benefit of analgesia}

The Overall Benefit of Analgesia Score (OBAS) will be assessed. ${ }^{19}$ The OBAS is a multidimensional seven-item instrument in which patients indicate (on a 0-4 scale, ranging from 'not at all' to 'very much') the level of current pain and distress arising from several symptoms such as itch. The OBAS is measured posthospitalisation (at home) at days 2 and 3 (study end).

\section{Analgesic dosage}

The total dosage of administered analgesics will be assessed during hospital stay and noted in the medical record. The total dosage of administered analgesic at home post operation will be assessed at days 2 and 3 (study end) by asking patients to indicate which pain medication they use/have used.

\section{Analgesic request by a patient}

Analgesic request will be assessed during hospital stay and noted in the medical record.

\section{Perceived empathy}

Perceived empathy will be determined using the Consultation and Relational Empathy Measure (CARE) ${ }^{20}$ in which the term 'doctor' is replaced with 'nurse' and 'consultation' is replaced with 'contact' (10 items, 1-5 scale ranging from 'poor' to 'excellent' (and 'not relevant'), eg, 'How was the nurse at showing care and compassion'). Perceived empathy is measured at day 3 (study end).

\section{Perceived expectation}

We will measure the extent to which participants thought nurses had induced the expectation that medication would be effective to decrease their pain. This will be assessed using a one-item self-created VAS scale ('no effect at all' to 'a lot of effect', ranging from 0 to 10). Perceived expectation will be measured at day 3 (study end).

\section{State anxiety}

Patients' level of anxiety will be measured by the Dutch 10-item State measure of the State-Trait Anxiety Inventory (STAI-state $)^{21}(1-4$ scale ranging 'not at all' to 'very much'). Patients' anxiety is assessed prehospitalisation, and at day 3 (study end).

\section{Mood}

Mood will be measured using the Positive and Negative Affect Schedule (PANAS) ${ }^{22}$ (20-items, 1-5 scale ranging from 'not at all' to 'very much', eg, 'I am exited' or 'I am upset'). Mood is measured prehospitalisation, and at day 3 (study end).

\section{Satisfaction}

Participants' satisfaction with the provided care by the nurses during day care will be assessed using a one-item self-created VAS scale ('not at all' to 'very much', 0-10 range). Satisfaction will be measured at day 3 (study end). 


\section{General pain evaluation}

Whether the pain following the operation has been better or worse than expected will be measured using a one-item self-created VAS scale (ranging from 'much worse than expected' to 'much better than expected', $0-10$ range). Pain evaluation is measured at day 3 (study end).

\section{General evaluations regarding hospitalisation}

Patients' evaluations of their hospitalisation are measured using two items: (1) how likely it is that the patient would recommend this hospital to other tonsillectomy patients (using an adapted item from the Consumer Quality Index (CQ Index $)^{23}$ (0-10 scale 'would definitely not recommend' to 'would definitely recommend') and (2) their overall rating of the quality of care provided by the hospital during hospitalisation, using an adapted item from the CQ Index ${ }^{23}$ (0-10 scale, 'very poor care' to 'extremely good care'). This is measured at day 3 (study end).

\section{Other outcomes}

The following background characteristics of patients are measured prehospitalisation:

\section{Sociodemographics}

For example, date of birth, gender, marital status, education, ethnicity, societal position and date of operation.

\section{Functional health status}

Measured using the Dartmouth COOP [from the Dartmouth Primary Care Cooperative Information Project known as the "CO-OP Project"] functional health assessment charts/World Organisation of National Colleges, Academies, and Academic Associations of General Practices/Family Physicians (COOP-WONCA); seven-item scale assessing several health status elements, for example, physical fitness, on a 1-5 scale ranging from 'not limited at all' to 'severely limited'.24 25

\section{General experiences/expectations/attitudes medications}

We will measure the extent to which patients generally (1) benefit from, (2) have positive expectations towards the effect of and (3) have objections against taking medicines. This will be done using self-created VAS scales (ranging from 'not at all' to 'very much', 0-10 range).

\section{General reporting of pain}

We will measure whether patients generally are inclined to report their pain using a self-created VAS scale (ranging from 'never' to 'always', 0-10 range).

\section{Attitudes towards operation}

The extent to which participants (1) are dreading, and (2) are afraid of the operation will be measured using two self-created VAS scales (ranging from 'not at all' to 'very much', 0-10 range).

In addition, we will measure:

\section{Data medical record}

We will ask patients' permissions to access the medical record. We will routinely use medical background data (diagnosis, weight and prescribed medication) and analgesic information (as aforementioned). If needed, additional data will be screened for (eg, in cases of outlier data the medical record might provide useful information).

\section{Background measures for nurses}

The following nurses' characteristics will be measured at study start.

\section{Sociodemographics}

For example, date of birth, gender and type of nurse (ie, nurse in training, regular nurse, specialised nurse).

\section{Empathy personality trait}

We will measure nurses' empathic abilities using the Interactive Reactivity Index (IRI). ${ }^{26}$ The IRI consists of 28 items (eg, 'I often feel sorry for people who are less fortunate than me') which are scored on a 1-5 scale (ranging from 'describes me not at all' to 'describes me very well').

An overview of the measured outcomes at different time points is provided in table 1 .

\section{Adherence to the communication manipulation protocol}

To verify the fidelity of the communication manipulation, the interaction between nurses and patients will be audio recorded. The adherence will be verified by listening back to a random sample ( $10 \%$ of the sample) of audio-recorded visits and to determine the adherence to the protocol (as is comparably done by Kaptchuk et $a l^{10}$ ). Two research assistants who are not otherwise involved in the study will independently evaluate the audio recordings on adherence to the protocol.

\section{Data analysis plan}

All data will be analysed using STATA 13.0 with two-sided significance testing at $\mathrm{p}<0.05$. All available data from patients will be included in the analysis and missing data might be imputed. An intention to treat analysis will be performed, thereby also examining selective attrition.

\section{Primary outcomes}

Descriptive statistics will be calculated for patients' reported pain intensity (main outcome measure) for the different time points during and post hospitalisation. Since our design consists of a two (expectancy: enhanced vs standard) by two (empathy: enhanced vs standard) design, all outcomes will be analysed using either analyses of variance (ANOVA) (if focused on a specific time point) or multilevel repeated-measures regression analyses (if focused on different time points which means that several ratings are included for one person). Both communication elements (ie, expectancy and empathy) are dummy coded. Main effects and interaction effects of expectancy and empathy will be explored. New insights gathered during the analysing process might be examined (if feasible). 


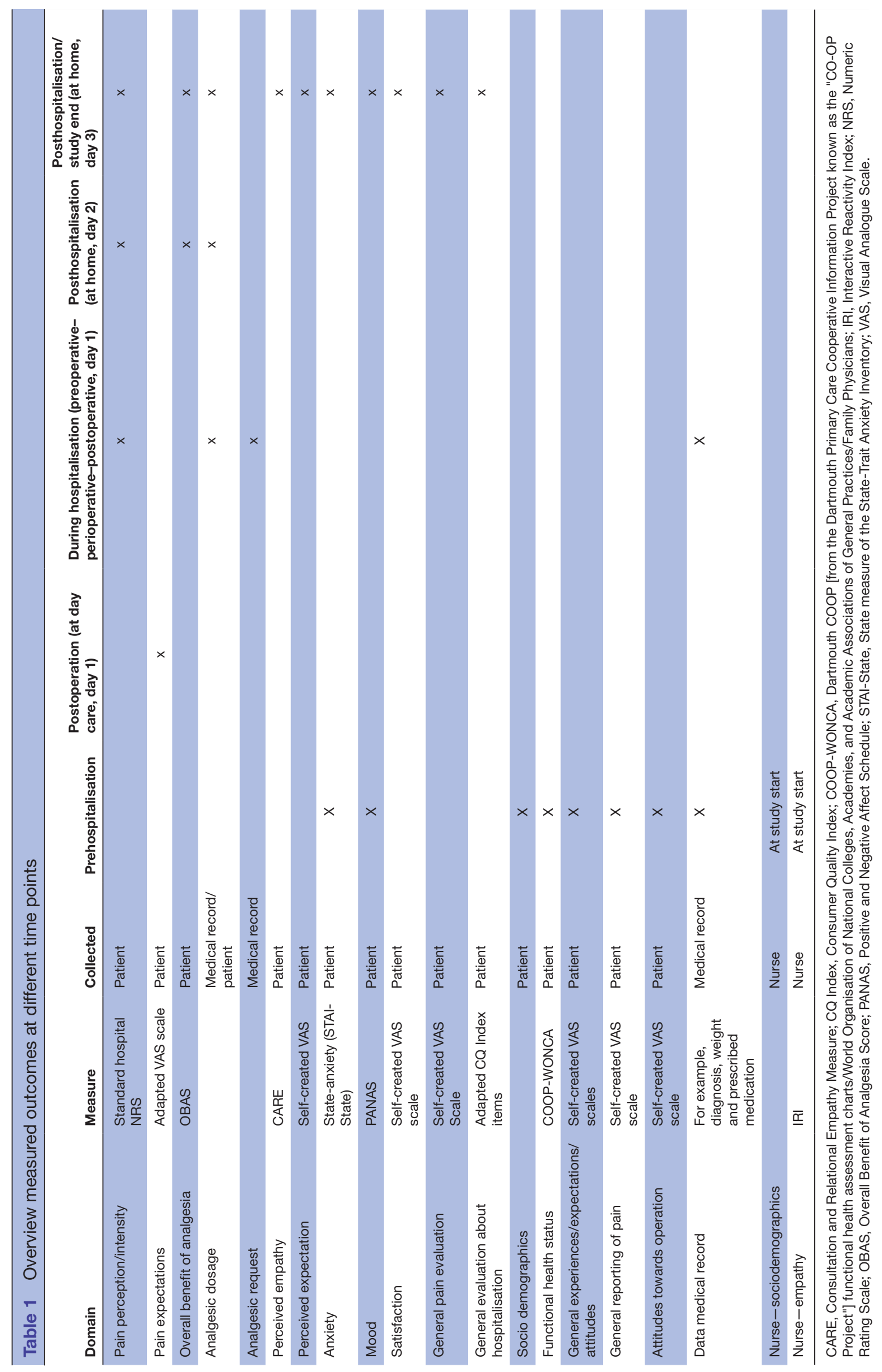




\section{Secondary outcomes}

Descriptive statistics will be calculated for secondary outcome measures. The effect of our manipulated variables will be analysed using analyses of variance.

\section{Other outcomes}

Frequencies and means will be calculated for the demographics. The four groups will be checked on equality by using chi-squared tests or ANOVA. If groups differ on specific variables, these variables might be used as control variables in the multilevel analysis.

\section{Adherence to communication protocol}

Data of the audio recordings are observed by trained coders on adherence to the protocol to verify fidelity. First, $10 \%$ of the audio recordings are independently checked on adherence to the protocol. For this purpose, the aforementioned main (verbal) features of the manipulations (see 'Intervention section') are described and rated for their occurrence when listening to the audio recordings. Using this, it is determined to which of the four conditions the audio recording belongs to. Second, inter-rater reliability between the coders of the outcomes will be assessed by calculating Cohen's kappa. Values ranging between 0.21 and 0.41 are considered fair, values between 0.41 and 0.60 are considered moderate and values greater than 0.61 are considered good (ie, substantial/ almost perfect). We consider values as reliable if Cohen's kappa is greater than $0.41 .{ }^{27}{ }^{28}$ Moreover, the number of nurse-patient interactions and duration of interactions is measured for each audio recording.

\section{ETHICS AND DISSEMINATION \\ Risks and burden for participants}

All participating patients receive usual care with regard to surgery, analgesia and preoperative and postoperative treatment. There are no risks associated with this clinical study. The communication manipulation will be provided on top of standard care and is designed in such a way that there will be no harmful effects for patients. Although practitioners' communication is deliberately manipulated and associated with both positive and less positive effects, the communication manipulation does not cross important norms or values of acceptable behaviour nor will it affect the psychological integrity of patients. Variations in nurse-patient interactions occur naturally within clinical settings, justifying our approach. Moreover, although patients are informed about the study by their treating clinicians, informed consent will be gathered by the research team. The clinical and research team will stress that participation is voluntary and will not affect standard clinical care. Patients are always free to withdraw their participation in the study. Finally, it can be a burden for patients to complete a few additional questionnaires. We attempt to decrease the burden by using short questionnaires and limiting follow-up to 3 days. Results, ultimately, will provide more insight into the effect of communication on patient outcomes.

\section{Adverse and serious adverse events}

All adverse events reported spontaneously by the patient or observed by the clinical or research team will be recorded. All serious adverse events (SAEs) will be reported to the ethical committee who has approved the study and the online database. This will be done within 15 days ( 7 days for the first reporting if an SAE resulted in death or was life threatening). Due to the content of the intervention, we do not expect SAE's to happen. The research team, supported by the clinical team, will regularly check the medical records for the occurrence of any adverse events and serious adverse events.

\section{Confidentiality}

Patients' data will be anonymised using an identification number. This code will be safeguarded by an independent contact person at NIVEL and this information will be kept on a protected drive using a protected file independently of the research data. The researchers involved in this study will have access to the research data. The audio recordings will not be destroyed after the research, but will be added to the NIVEL audio/video database. At present, NIVEL has a database of around 18000 (digitised) video-recorded and audio-recorded healthcare visits and a well-equipped infrastructure with computerised observation units.

\section{Ethics and dissemination}

At the minimum, the results of this study will be published in international peer-reviewed scientific journals. A lay summary of the results will be published as well and sent to participants if they are interested.

\section{DISCUSSION}

This innovative study aims to manipulate communication to determine how expectations and empathy can lead to placebo effects and help minimise patients' postoperative pain (among other outcomes). The results can help to shed more light on how communication can be used alongside medical care to enhance patients' outcomes for the better.

That being said, manipulating communication in clinical care poses methodological, ethical and logistical challenges. The success of the study will depend on the success of the delivery of the manipulations. To ensure communication differs between the various groups, and to avoid contamination, all nurses have been trained and the research team is available (onsite and offsite) for questions, practice and feedback. On most intervention days, a member of the research team is present at the day care ward. This is much appreciated by nurses, and ensures that the appropriate manipulation is often practiced before a patient is admitted. Throughout an intervention day, 
the signposting of all systems (ward lists/hard copy patient records) and the appropriate pocket cards with examples also serve as a constant reminder of the group allocation. The success of the manipulation is checked using the audio recordings of nursing interactions and will assist in interpreting our results. While varying communication, the ethical boundaries of not providing any suboptimal communication are and will be clearly adhered to and are stressed in contacts with involved healthcare professionals. Finally, patients come in contact with many clinicians before and during hospitalisation. Informing all clinicians and ensuring all but the day care ward nurses will standardise their communication is crucial to ensure causal effects of the manipulated communication can be determined. Therefore, both the research and clinical team involved have ensured many contact moments with clinicians to personally inform them about the study and appropriate information material has been circulated at study start.

Of course, this study is beforehand not without limitations. Most importantly, due to the clinical nature of the study, it is impossible to standardise communication elements beyond expectancy and empathy. We did, however, instruct nurses to vary only the manipulated communication and keep the remaining care and communication standard. We, therefore, believe these elements to not differ widely, but if evenly, between conditions. Moreover, time differences and differences in the number of nurse-patient interactions between the conditions could potentially occur. We did, however, tried to ensure that the manipulations differed as minimally as possible in time (eg, enhanced empathy consists of little time-consuming behaviours such as sitting opposed to standing) and all interventions need to be delivered within nurses' standard work time. Moreover, we instructed nurses to display the manipulations during all their standard interactions, and did not instruct them to have extra interactions in the enhanced conditions. We, therefore, believe to have minimised the risk for time differences and interaction differences to occur between conditions. For the $10 \%$ checked audio recordings, consultation time and the number of nurse-patient interactions are measured, which might help us in interpreting the findings. We acknowledge it remains a limitation of this complex clinical study that due to focus and power constraints we will not completely measure or control for all variables beyond, and time/interaction differences between, the manipulations. A last limitation is that nonverbal behaviour (eg, eye contact) cannot be taken into account when determining adherence to the protocol using the audiotapes.

Despite these challenges and limitations, we believe that this study is of utmost importance to bring the field of communication and pain research forward. Without conducting controlled studies into the effect of communication, communication will always remain a soft-sided add-on. In order to overcome this, we would recommend future studies to also include biological and clinical outcomes. Most importantly, we hope that this study and detailed protocol will provide an impetus for further work in this important area turning communication from 'art to science'.

\section{Author affiliations}

${ }^{1}$ Department of Communication, NIVEL (Netherlands Institute for Health Services Research), Utrecht, The Netherlands

${ }^{2}$ Department of Primary and Community Care, Radboud University Medical Center, Nijmegen, The Netherlands

${ }^{3}$ Faculty of Health Sciences, Science Centre Health and Technology, University College of Southeast Norway, Drammen, Norway

${ }^{4}$ Department of Anesthesiology, OLVG Hospital, Amsterdam, The Netherlands ${ }^{5}$ Department of ENT, OLVG Hospital, Amsterdam, The Netherlands

Acknowledgements We thank Peter Spreeuwenberg for his help with the data analysis plan and power calculation. We thank the clinicians working at the daycare ward, the operation theater, the PACU and at the ENT and Anesthesia department for their help in facilitating the study logistics. We especially thank the daycare ward nurses for delivering the intervention.

Contributors JMB is the Chief Investigator. JMB developed the initial concept of the study, supported by SvD and LMvV. LMvV, JMB, SvD and SI led on the protocol development, while all authors provided input for the trial design and protocol. $M B G, B T$ and GWvD led on the standardisation of hospital routine, pain protocol and medical content. LMvV and SI (as of August 2016 also supported by MK, while SI left the project in 2017) lead on the daily management of the trial, supported by MBG, BT, GWvD, SvD and JMB. All authors have read, contributed and approved the final manuscript.

Funding The study is funded by the Spinoza Prize from the Dutch Research Council awarded to JMB. LMvV is partly funded by a grant from the Foundation for the Science of the Therapeutic Encounter (F-STE). The funder had no role in the study design and decision to submit the report for publication. The funder will have no role in the collection, management, analysis and interpretation of data or writing and publication of future publications.

Competing interests None declared.

Ethics approval This study has received ethical approval from the ethical committee of the UMC Utrecht (number 16/144, NL55225.041) and the institutional review board of the participating hospital (number WO 16.506). All amendments will be notified to the ethical committeethat gave a favourable opinion.

Provenance and peer review Not commissioned; externally peer reviewed.

Open Access This is an Open Access article distributed in accordance with the Creative Commons Attribution Non Commercial (CC BY-NC 4.0) license, which permits others to distribute, remix, adapt, build upon this work non-commercially, and license their derivative works on different terms, provided the original work is properly cited and the use is non-commercial. See: http://creativecommons.org/ licenses/by-nc/4.0/

(c) Article author(s) (or their employer(s) unless otherwise stated in the text of the article) 2017. All rights reserved. No commercial use is permitted unless otherwise expressly granted.

\section{REFERENCES}

1. Miller FG, Kaptchuk TJ. The power of context: reconceptualizing the placebo effect. J R Soc Med 2008;101:222-5.

2. Finniss DG, Kaptchuk TJ, Miller F, et al. Biological, clinical, and ethical advances of placebo effects. Lancet 2010;375:686-95.

3. Turner JA, Deyo RA, Loeser JD, et al. The importance of placebo effects in pain treatment and research. JAMA 1994;271:1609-14.

4. Kaptchuk TJ, Miller FG. Placebo Effects in Medicine. N Engl J Med 2015;373:8-9.

5. Tracey I. Getting the pain you expect: mechanisms of placebo, nocebo and reappraisal effects in humans. Nat Med 2010;16:1277-83. 
6. Mistiaen $\mathrm{P}$, van Osch $\mathrm{M}$, van Vliet $\mathrm{L}$, et al. The effect of patientpractitioner communication on pain: a systematic review. Eur $J$ Pain 2016;20:675-88.

7. Benedetti F, Maggi G, Lopiano L, et al. Open versus hidden medical treatments: The patient's knowledge about a therapy affects the therapy outcome. Prevention \& Treatment 2003;6:1a.

8. Charlesworth JEG, Petkovic G, Kelley JM, et al. Effects of placebos without deception compared with no treatment: A systematic review and meta-analysis. J Evid Based Med 2017;10:97-107.

9. Jubb J, Bensing JM. The sweetest pill to swallow: how patient neurobiology can be harnessed to maximise placebo effects. Neurosci Biobehav Rev 2013;37:2709-20.

10. Kaptchuk TJ, Kelley JM, Conboy LA, et al. Components of placebo effect: randomised controlled trial in patients with irritable bowel syndrome. BMJ 2008:336:999-1003.

11. Bensing JM, Verheul $\mathrm{W}$. The silent healer: the role of communication in placebo effects. Patient Educ Couns 2010;80:293-9.

12. Verheul W, Sanders A, Bensing J. The effects of physicians' affectoriented communication style and raising expectations on analogue patients' anxiety, affect and expectancies. Patient Educ Couns 2010;80:300-6.

13. van Osch $M$, van Dulmen $S$, van Vliet $L$, et al. Specifying the effects of physician's communication on patients' outcomes: A randomised controlled trial. Patient Educ Couns 2017;100:1482-9.

14. Manias E, Botti M, Bucknall T. Observation of pain assessment and management--the complexities of clinical practice. J Clin Nurs 2002;11:724-33.

15. Breivik $\mathrm{H}$, Borchgrevink PC, Allen SM, et al. Assessment of pain. Br J Anaesth 2008:101:17-24.

16. Nederlandse Vereniging voor Anesthesiologie [Dutch Association for Anaesthesiology]. Richtlijn Postoperatieve pijn [Guidline for postoperative pain]. Utrecht, The Netherlands, 2012.

17. Petersen GL, Finnerup NB, Grosen K, et al. Expectations and positive emotional feelings accompany reductions in ongoing and evoked neuropathic pain following placebo interventions. Pain 2014;155:2687-98.
18. Devilly GJ, Borkovec TD. Psychometric properties of the credibility/expectancy questionnaire. J Behav Ther Exp Psychiatry 2000;31:73-86.

19. Lehmann N, Joshi GP, Dirkmann D, et al. Development and longitudinal validation of the overall benefit of analgesia score: a simple multi-dimensional quality assessment instrument. $\mathrm{Br} \mathrm{J}$ Anaesth 2010;105:511-8.

20. Mercer SW, Maxwell M, Heaney D, et al. The consultation and relational empathy (CARE) measure: development and preliminary validation and reliability of an empathy-based consultation process measure. Fam Pract 2004;21:699-705.

21. Spielberger CD, Gorsuch RL, Lushene R, et al. State-trait anxiety inventory for adults: sampler set: manual, test, scoring key. Palo Alto, CA: Mind Garden Consulting Psychologists Press, 1983.

22. Watson D, Clark LA, Tellegen A. Development and validation of brief measures of positive and negative affect: the PANAS scales. $J$ Pers Soc Psychol 1988;54:1063-70.

23. van Kessel $P$, Hendriks M, van der Hoek L, et al. CQ Index Chronisch Hartfalen. Ontwikkeling van de vragenlijst en de ervaringen van mensen met chronisch hartfalen met de ziekenhuiszorg[CQ Index Chronic heart failure. Development of the questionnaire and the experiences of chronic heart failure patients with hospital care]. Utrecht, the Netherlands: NIVEL, 2015.

24. Van Weel C. Functional status in primary care: COOP/WONCA charts. Disabil Rehabil 1993;15:96-101.

25. Van Weel C, Konig-Zahn C, Touw-Otten F, et al. Measuring functional status with the COOP/WONCA Charts. A manual. Groningen, the Netherlands: Noordelijk Centrum voor GEzondheidsvraagstukken (NCG)/Northern Centre of Health Care Research (NCH), 2012.

26. Davis MH. Measuring individual differences in empathy: Evidence for a multidimensional approach. J Pers Soc Psychol 1983;44:113-26.

27. Sim J, Wright CC. The kappa statistic in reliability studies: use, interpretation, and sample size requirements. Phys Ther 2005:85:257-68.

28. Landis JR, Koch GG. The measurement of observer agreement for categorical data. Biometrics 1977;33:159. 